Als Endresultat ergibt sich, daß die meisten Probleme, die mit den genetischen Beziehungen der Elemente und mit ihrem Aufbau zusammenhängen, wie sie uns die Lehre von der Radioaktivität und das periodische System in seiner neuen Form exschlossen hat, zu ihrer völligen Klärung im höchsten quantitativen Sinne voraussichtlich noch auf lange Zeit der Beihilfe feinster chemischer Präzisionsarbeit bedürfen werden.

\section{Über die Massenverteilung im Erdinneren, verglichen mit der Struktur gewisser Meteoriten.}

Von V. M. Goldschmidt, Kristiania.

Die Ergebnisse der Seismologie lehren uns bekanntlich, daB im Erdinnern eine Schalenanordnung verschieden dichter Stoffe angenommen werden muB, derart, daB um einen schweren Erdkern nach außen hin immer leichtere Stoffe angeordnet sein müssen. Utber den stofflichen Bestand der einzelnen Schalen wissen wir, daß die äußerste Erdkruste aus verhältnismä日ig leichten Silikaten besteht, der Erdkern hingegen höchstwahrscheinlich aus Nickeleisen. Für die dazwischen liegenden Schalen nimmt man gemeinhin an, daß diese vorzugsweise aus Magnesiumsilikaten bestehen, eventuell gemischt mit Nickeleisen.

Ich möchte annehmen, daß man, gestützt auf neuere Engebnisse der Petrographie, zu wahrscheinlicheren Annahmen über die Beschaffenheit dieser Schalen gelangen kann.

Die Zusammensetzung der äuBersten festen Schale, der Silikathülle, kennen wir mittels Durchschnittzahlen aus den Analysen frischer Eruptivgesteine, wie sie beispielsweise zuletzt von H. S. Washington berechnet worden sind. Die Dichte dieser Silikathülle können wir zu etwa 2,8 veranschlagen, in Übereinstimmung mit der Dichte des Opdalits ${ }^{1}$. Die Dicke dieser Schale wird auf etwa $120 \mathrm{~km}$ geschätzt, nach den Ergebnissen über die Ausgleichstiefe der Schwereanomalien.

Unter dieser Silikathülle wird gewöhnlich eine schmelzflüssige Schale basischer Silikate angenommen. Neuere petrographisobe Arbeiten, insbesondere $P$. Eskolas wichtige Arbeiten über die Eklogite machen es mir indes wahrscheinlicher, dab diese Schale ans festen Silikatgesteinen in einem Zustande besonderer Kompression besteht, einem Zustande, in welchem die jeweilig dichtesten Minerale und Mineralkombinationen vorliegen.

1) Der Opdalit ist ein vom Verf. entdeaktes Tiefengrastrin, welches in seiner ohemischen Zusammensetzung sehr nahe der Durchscibnittsziasammensetzung der SiliWathotlue entspricht. Er besteht aus Plagioklas (Andesin, etwo $43 \%$ ), Kalifeldspat (etwa 14\%), Biotit (etwa $10 \%$ ), diopsidischem Augtit (etwa $5 \%$ ), Hypersthen (etwa $9 \%$ ), Quarz (etwa 15\%) sowie ca. $2 \%$ Erzen und Apatit.
Eklogitgesteine von ungefähr gabbroider $\mathrm{Zu}$ sammensetzung besitzen eine Dichte von etwa 3,6 , bei Druckentlastung gehn sie in Gabbrogesteine: bzw. Gabbroschmelzflüsse über, mit einer Dichte von etwa 3. Die Möglichkeit isostatischen Ausgleichs an der Grenzfläche zwischen Silikathülle und Eklogitschale ist durch die bedeutende Volumenänderung beim Ubergang gegeben. Bei lokaler oder regionaler Zunahme des Belastungsdruckes nimmt unter der Druckstelle die Menge. der im Eklogitzustand befindlichen Substanz zu, bei Druckentlastung wird der Eklogit in leichtere Silikate oder Silikatschmelzflüsse verwandelt. Druckentlastung ohne gleichzeitige Temperaturerniedrigung kann nämlich innerhalb eines gewissen Temperaturintervalles zur Schmelzung von Eklogit unter Entstehung von Basaltmagma fuldren. Es ist nieht unwahrscheinlich, dab der Herd. vulkanischer Erscheinungen in manchen Fälen eben an der Grenze zwischen Silikathülle und. Eklogitschale zu suchen ist. Fierfür spricht das

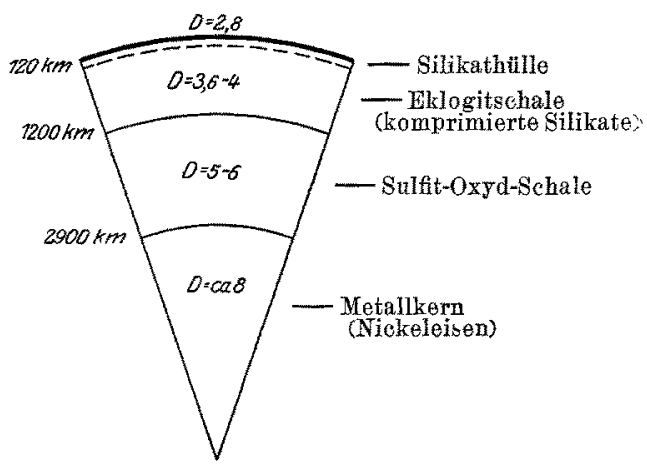

Fig. 1. Durchschnitt durch die Erde.

Auftreten von Eklogiteinschlüssen beispielsweise in den Kimberlitdurchbrüchen in Südafrika').

Die Eklogitschale ist die normale Heimat einer Reihe spezifisch schwerer Minerale, die nur ausnahmsweise in höheren Teilen der Erdrinde entstehn können, wie besonders Pyrop, jadeitische und chloromelanitische Pyroxene sowie Diamant.

Die Eklogitschale dürfte bis zu einer Tiefe von etwa $1200 \mathrm{~km}$ hinabreichen ${ }^{3}$ ), in dieser Tiefe trifft man eine Unstetigkeitsfläche gegen eine tiefere und vermutlich wesentlich schwerere Schale. Man hat in der Regel angenommen, daß in den tieferer Teilen der Steinschalen eine zunehmende Beimengung matallischen Eisens (bzw. Nickeleisens) auf-

2) Solche Eklogitleinschlüisse in Explosionsschloten dürften wohl der Eklogitschale entstammen, hierfiur spricht neben der Art des geologtisehen Auftretens be sonders auch das Vorkommen das Diamanten. Dile meisten anderen Eklogitworkommen, idhe uns zugänglich sind, stammen jedoch nicht aus der Eklogitschale, sondiern sind lokalle Bildungen in hülle, entistanden durch lokale Drucksteigenung in Faltungsregionen, wi:e beispielsweise die alpinen Eklogitgesteine.

$\left.{ }^{3}\right)$ In den tieferen Teilen dier Eklogitschale duirfte die Dichte bis ca. 4 ansteigen. 
tritt. Bei dem groben Dichteunterschied zwischen den schwersten gesteinsbildenden Silikaten einerseits (Dichte $3,6-4$ ), Nickeleisen anderseits (Dichte 7,6-8) halte i.ch es nicht für wahrscheinlich, daß in dem starken Schwerefeld der Erde eine solche Mischung bestandfähig wäre, wenigstens nicht in Gesteinen, die zeitweilig in schmelzflüssigem Zustande gewesen sind. Ich möchte deshalb annehmen, daß unter den tiefsten Teilen der einigermaßen reinen Silikatschalen nicht eine Mischung von Silikaten und Eisen auftritt, sondern solche Stoffe, die einerseits schwerer als die Silikate sind, anderseits leichter als der eigentLiche Eisenkern. Derartige Stoffe sind insbesondere gewisse sulfidische und oxydische Erze. Ich möchte deshalb glauben, daß unterhalb der Eklogitschale, mehr oder wenigex scharf abgegrenzt gegen diese, eine Schale von Sulfiden und Oxyden auftritt. Diese dürfte vorwiegend aus Schwefeleisen und Magnetit bestehen, daneben dürfte sie Chromit, Titaneisen und Rutil enthalten sowie kleinere Mengen noch anderer Sulfide als nur die des Eisens. Die Bildung dieser" Schale dürfte teilweise durch Entmischung in flüssigem Zustande, teilweise durch Absinken foster oxydischer Erze brw. flüssiger Sulfidmagmen aus dem Steinmante]' vor sich gegangen sein. Ob diese SulfidOxyd-Schale in sich homogen ist oder selbst wieder eine Unterteilung nach der Dichte aufweist, erscheint noch ungewib, doch ist letzteres wahrscheinlicher; ihre mittlere Dichte dürfte etwa gleich 5-6 zu setzen sein. Eine Schale aus "Eisenerzen" wurde bereits von W. Klufmann angenommen. Diese Schale reicht nach seismologischen Daten bis zu einer Tiefe von etwa 2900 Kilometern, ach unten folgt dann der eigentliche Erdkern, der höchstwahrscheinlich aus Nickeleisen mit einer Dichte gleich etwa 8 besteht.

Ein derartiger Schalenbau des Erdballs ist un umgänglich, falls idie Erde ursprünglich schmelzflüssig gewesen ist, da ein Gemenge von Silikaten, Sulfiden und freiem Eisen in einem großen Temperaturgebiete oberhalb des Schmelzgebietes in drei unmischbare Flüssigkeiten zerfällt. Die Sonderung des silikatischen Anteils in eine äubere Hïlle und eine innere Eklogitschale ist hingegen an eine mehr oder weniger vollständige Kristallisation geknüpft, während welcher Kristallisation auch noch eine Zunahme der Sulfid-Oxyd-Schale auf Kosten solcher Sulfide und Oxyde stattgefunden hat, die aus der kxistallisierenden Silikatschale abgesunken sind.

Die relative Seltenheit der meisten technisch wichtigen Schwermetalle in der uns zugänglichen äuBeren Silikathülle beruht nun offenbar darauf, daß bei der ersten Trennung des Erdkörpers in Metallkern, Sulfidschale und Silikatschale alle diejenigen Metalle, die leichter reduzierbar sind als Eisen oder besonders starke Affinität zum Schwefel besitzen, von vornherein vorzugsweise im Metallkern und in der ursprïnglichen Sulfidschale angereichert wurden, so dab nur ein ganz kleiner Bruchteil dieser Metalle in der Silikatschale verblieb. Auch von diesem kleinen Bruchteil sind bei der späteren Abkühlung der Silikatschale gewiß ganz bedeutende Mengen durch Absinken in die tieferen Teile des Erdkörpers gewandert. Diose Art der Verteilung von edlen und halbedlen Metallen zwischen Metallkern, SulfidOxyd-Schale und Silikatschale entspricht durchaus den metallurgischen Trennungsoperationen") bei der Bildung von ,Schlacke", "Stein". und "Eisensau". H. S. Washington hat die chemischen Elemente ebenfalls in "metallogenetische" und ,petrogenetische" eingeteilt, nach ihrem Auftreten teils in der silikatischen Hulle, teils in den metallischen Kernregionen.

Eine notwendige Vorbedingung für die Entstehung eines solchen konzentrischen Schalenbaues, wie er dem Frdbau zugrunde liegt, ist das Vorhandensein eines genügend starken Schwerefeldes, wie es etwa durch die eigene Masse der Erde hervorgerufen wird. Bei kleineren kosmischen Massen mag in vielen Fallen das Schwerefeld ungenügend sein, um eine solche, relativ scharfe räumliche Sonderung zu bewirken. Ich möchte glauben, daß manche Meteoriten, insbesondere die Pallasite, instruktive Beispiele für diese Erscheinung bieten.

In den meisten Pallasiten bilden die Silikate (vor allem der Olivin) rundliche Einschlüsse im Nickeleisen, die offenbar in der Weise entstanden sind, daß flüssige Silikattropfen in einem Metallschmelzflusse suspendiert waren. Eine solche Suspension. wäre in starken Schwerefeldern äuBerst unbeständig; das System würde sich in Metallschmelze und Silikatschmelze trennen. Dasselbe gilt für eine Nickeleisenschmelze mit darin suspendierten festen Sillkatkörnern. Nur durch sehr schnelle Erstarrung könnte ein solcher instabiler Zustand im irdischen Schwerefeld arretiert werden, nun zeigt aber die Struktur des Eisenanteils in Pallasiten keinerlei Merkmale sehr plötzlicher Kristallisation ${ }^{5}$. Wir werden deshalb zu der Folgerung geführt, daß die Pallasite zur Zeit ihrer Erstarrung sich nicht in einem starken Schwerefelde befunden haben, daß sie also offenbar einem relativ kleinen Himmelskörper angehört haben und und nicht feste Bruchstücke eines bereits erstarrten großen Körpors sein können, falls sie

4) Aug den Betrachtungen, in denen ich oben die Entistehungsweise des Schallenbaus im Erdlkörper dargelegt habe, enstieht man, ¿daß dive Silikathälle der Eride systematisch ihres Inhalts an manchen wertwollen Meballen berambt worden ist, ersteng durch die anginstige Primärverteilung dieser Metalle zwischen Silikatschmelze, Sulfildschmelze und Metallischmelze, zweitens durich späteres Absinken bei der Kristallisation. Jetzterer Umistand hat insbesondere solche spezifisch schworen Metalle getroffen, die leicht reduzierbar sind whid einen hohen Sohmelzpunkt besitzen (ralso frïhzeitig arts dier Silikatschmelze auskristallisieren). Die Seltenheit des Platins in den Gestielimen der Silikathïlle durfte bierin begrundet sein.

5) Die Struktur des Eisenanteils deutet im Gegenteil auf eine sehr Jamggrame Kristallisation. 
nicht etwa einer späteren Umschmelzung unterworten worden sind. Thre Erstarrungsgeschichte hat sich jedenfalls ohne Gegenwart eines starken anisotropen Schwerefeldes abgespielt.

Im selben Sinne, wenngleich nicht derart kraß, kann das Auftreten von Troilit- (und Troilit-Ohromit-) Knollen in meteorisehem Nickeleisen gedeutet werden. Diese Knollen entsprechen der Sulfid-Oxyd-Schale im Erdinnern.

Es wäre von grobem Interesse, die Gesamtheit der Meteoriten auf den Grad ihrer Schweresonderung zu untersuchen, da man hieraus vielleicht $z u$ weiteren Aufschlüssen über ihre Herkunft und Geschichte gelangen könnte.

In manchen Eisenmeteoriten findet man ungleichmäBige Verteilung der leichteren Einschlüsse im Nickeleisen, wie lokale, unsymmetrische Anreicherung von Olivinkristallen, schlierige oder streifenformige Anordnung des Schwefeleisens, welches darauf hindeutet, daB gewisse, wenn auch schwache Schwerewirkungen ihren Einfluß geltend machten, während das Nickeleisen noch flüssig war ${ }^{6}$ ).

Von besonderem Interesse wäre es auch, die Erstarrungsgeschichte der Pallasite näher zu studieren, um insbesondere die Frage zu behandeln, in welchen Pallasiten die Silikattropfen bereits vor Erstarrung des Metallbades erstarrten, in welchen erst nachher, und ob diese Erstarrungsgeschichte in Beziehung zu den Schmelzdiagrammen Nickel-Eisen und Olivin-Fayalit gebracht werden kann.

In Meteoriten finden wir niemals Mineralkombinationen, welche der Eklogitschale der Erde entspreehen würden; dies hat offenbar seinen Grund darin, daß in Kleinen Himmelskörpern nicht der notwendige Belastungsdruck für die Bildung von Eklogiten aufgebracht werden kann.

Das Vorkommen von Diamant als Seltenheit in einzelnen Meteoreisen könnte zwar auf Bildungsbedingungen hindeuten, welche mit der Eklogitfacies vergleichbar sind, immerbin kann der Diamant auch ohne starken Belastungsdruck entstanden sein, in Analogle mit Moissans bekannter Synthese.

Das Auftreten von solchen Struktureigentümlichkeiten, die auf Abwesenheit starker Gravitationswirkungen hindeuten, bringt uns zu der Frage, ob es experimentell' gelingen könnte, solche Strukturen nachzuahmen, wie sie den Pallasiten zu eigen sind. Im Laboratorium oder im technischen Schmelzofen sind die Einwirkungen des Schwerefeldes nicht zu eliminieren, wir können keine dauernde Suspension von Silikattropfen in geschmolzenem Nickeleisen herstellen; es beruht ja die technische Herstellung von Eisen im Hochofen gerade auf der gravitativen Sonderung von Eisen und Silikatschlacke. Es bietet sich nur eine Möglichkeit, den EinfluB eines Schwerefeldes (bzw. Trügheitsfeldes) bei einem Schmelzversuch

\footnotetext{
6) Möglicherweise durch Rotation des Meteoriten (Zentrifugalkraft) hervargebracht.
}

weitgehend auszuschalten, nämlich den Versuch in einer frei fallenden Schmelzmasse auszufuhren, die genügend schwer ist, um die Hemmung durch Luftwiderstand weitgehend auszuschalten, etwa indem man die Schmelze in einen granatenförmigen Behälter von einem Flugzeug himabfallen liebe.

\section{Neue physiologische Untersuchungen über das Leben in den Anden.}

\author{
Fon A. Loewy, Berlin.
}

In der Nummer vom 29. Juli d. J. der englischen Zeitschrift Nature findet sich ein Aufsatz des bekannten Cambridger Physiologen J. Barcroft: ,The physiology of life in the Andes."

Er enthält in Kurzer Darstellung die Ergebnisse von Untersuchungen, die in den peruanischen Anden angestellt wurden. Sie betreffen die Besonderheiten im Ablauf der physiologischen Vorgänge, welche ein beschwerdefreies und körperliche Árbeit gestattendes Leben in jenen Hochregionen für die Eingeborenen ermöglichen, und die Anpassungsvorgänge, die bei Tiefländern sich allmählich ausbilden, wenn sie längere Zeit in diesen Hohen leben, und die dazu fuhren, dab auch diese, wenigstens bei Körperruhe, schließlich keinerlei Krankheitssymptome mehr zeigen.

Beim Lesen des Barcroftschen Berichtes muB denjenigen, der selbst in den Zeiten von Deutschland's Glanz und Ruhm an ähnlichen Unternehmungen sich beteiligt hat, ein Gefühl der Niedergesehlagenheit und des Neides beschleichen bei der Wahrnehmung, daB deutsche Eorscher heute und wohl noch für lange Zeit auBerstande sind, derartige Untersuchungen auch nur zu planen, während die angelsächsisehen Forscher schon kurz mach Beendigung des Weltkrieges in der Lage waren, an große wissenschaftliche Unternehmungen heranzugehen und Gegenstände weiterzufördern, an deren bisheriger Bearbeitung die deutsche Wissenschaft einen nicht geringen Anteil hatte.

Aus der Darstellung Barcrofts geht dieser Anteil allerdings nicht hervor, denn sie beschränkt sich auf die Beschreibung der eigenen Expedition und deren Ergebnisse. Letztere bestätigen vielfach mur die älteren Befunde. In einigen Punkten jedoch bringen sie neue und überraschende Kenntnisse.

Mit Unterstutzung verschiedener englischer und amerikanischer Institute und mit Hilfe von Spenden von privater Seite bildeten sich eine englische und eine amexikanische Abteilung, deren erste von Liverpool, deren zweite ron New York nach Lima aufbrach. Von dort ging die Reise mit der transandinischen Bahn über die Randkordillere in Höhe von $5300 \mathrm{~m}$ hinab nach Oroya $(4000 \mathrm{~m})$, das in den Steppen zwischen den beiden parallelen Kordillerenketten gelegen ist, und ron da nach dem Bestimmungsorte Cerro de 